\title{
Surface-based morphometry study of the brain in benign childhood epilepsy with centrotemporal spikes
}

\author{
Zhengzhen Li ${ }^{1 \#}$, Jingjing Zhang ${ }^{1 \#}$, Fuqin Wang ${ }^{1}$, Yang Yang ${ }^{1}$, Jie Hu ${ }^{1}$, Qinghui Li ${ }^{1}$, Maoqiang Tian ${ }^{2}$, \\ Tonghuan $\mathrm{Li}^{3}$, Bingsheng Huang ${ }^{4,5}$, Heng Liu ${ }^{1}$, Tijiang Zhang ${ }^{1}$ \\ ${ }^{1}$ Department of Radiology, Affiliated Hospital of Zunyi Medical University, Medical Imaging Center of Guizhou Province, Zunyi, China; \\ ${ }^{2}$ Department of Pediatrics, Affiliated Hospital of Zunyi Medical University, Zunyi, China; ${ }^{3}$ Department of Neurological Rehabilitation of Children, \\ Affiliated Hospital of Zunyi Medical University, Zunyi, China; ${ }^{4}$ Medical AI Lab, School of Biomedical Engineering, Health Science Center, \\ Shenzhen University, Shenzhen, China; ${ }^{5}$ Shenzhen University Clinical Research Center for Neurological Diseases, Shenzhen, China \\ Contributions: (I) Conception and design: H Liu, T Zhang, B Huang; (II) Provision of study patients: M Tian, T Li; (III) Collection and assembly \\ of data: Z Li, J Zhang, F Wang, Y Yang, J Hu, Q Li; (IV) Data analysis and interpretation: B Huang, H Liu, T Zhang; (V) Manuscript writing: All \\ authors; (VI) Final approval of manuscript: All authors. \\ \#These authors contributed equally to this work. \\ Correspondence to: Dr. Heng Liu; Dr. Tijiang Zhang. Department of Radiology, Affiliated Hospital of Zunyi Medical University, Medical Imaging Center \\ of Guizhou Province, 149 Dalian Road, Huichuan District, Zunyi, China. Email: zmcliuh@163.com, tijzhang@163.com; Dr. Bingsheng Huang. Medical \\ AI Lab, School of Biomedical Engineering, Health Science Center, Shenzhen University, Shenzhen, China. Email: huangb@szu.edu.cn.
}

Background: The study aimed to explore cortical morphology in benign childhood epilepsy with centrotemporal spikes (BECTS) and the relationship between cortical characteristics and age of onset and intelligence quotient (IQ).

Methods: Cortical morphometry with surface-based morphometry (SBM) was used to compare changes in cortical thickness, gyrification, sulcal depth, and fractal dimension of the cerebral cortex between 25 BECTS patients and 20 healthy controls (HCs) with two-sample $t$-tests $[\mathrm{P}<0.05$, family-wise error (FWE) corrected]. Relationships between abnormal cortical morphological changes and age of onset and IQ, which included verbal intelligence quotient (VIQ), performance intelligence quotient (PIQ), and full-scale intelligence quotient (FIQ) were investigated with Spearman correlation analysis $(\mathrm{P}<0.05$, uncorrected).

Results: The BECTS patients showed extensive cortical thinning predominantly in bilateral frontal, temporal regions, and limbic system. Cortical gyrification increased in the left hemisphere and partial right hemisphere, and the decreased cortical gyrification was only in the left hemisphere. The increased sulcal depth was the left fusiform gyrus. There are no statistically significant differences in the fractal dimension. Correlation analysis revealed the negative correlation between age of onset and cortical thickness in the right precentral gyrus. It also revealed the negative correlation between the age of onset and cortical gyrification in the left inferior parietal gyrus. Also, there was negative correlation between VIQ and cortical gyrification in the left supramarginal gyrus of BECTS patients.

Conclusions: This study reveals aberrant cortical thickness, cortical gyrification, and sulcal depth of BECTS in areas related to cognitive functions including language, attention and memory, and the correlation between some brain regions and VIQ and age of onset, providing a potential marker of early neurodevelopmental disturbance and cognitive dysfunction in BECTS.

Keywords: Rolandic epilepsy; children; magnetic resonance imaging; surface-based morphometry (SBM); intelligence quotient (IQ)

Submitted Jul 02, 2020. Accepted for publication Sep 04, 2020.

doi: $10.21037 / \mathrm{atm}-20-5845$

View this article at: http://dx.doi.org/10.21037/atm-20-5845

(c) Annals of Translational Medicine. All rights reserved. 


\section{Introduction}

Benign childhood epilepsy with centrotemporal spikes (BECTS), also called Rolandic epilepsy, or self-limited epilepsy with centrotemporal spikes according to 2017 International League Against Epilepsy (ILAE) seizure classification (1). BECTS accounts for 8-25\% of epilepsy in children $(1,2)$, most of the age of onset is $3-13$ years, and the peak period (3) is $8-10$ years. During nocturnal sleep, seizures characterize it with centrotemporal spikes in the electroencephalogram $(2,4)$.

Although BECTS typically displays an excellent prognosis with remission of seizures before adolescence, some prevenient neuroimaging studies revealed abnormalities of brain structure and cognitive function in patients with BECTS, which affect the academic and future career development of children on some degree (5-9). The earlier studies mainly reported the abnormal cortical thickness and volume (5,6,10-13). Furthermore, considering the influence of antiepileptic drugs and the range of patients' age, the results require further validation.

Surface-based morphometry (SBM) offers more information for brain structural analysis, which might not be captured by voxel-based morphometry (VBM). Cortical characteristics acquired not only cortical thickness but also gyrification, sulcal depth, and fractal dimension associated with cognitive dysfunction and pathological changes in multiple neuropsychiatric disorders (14-17).

Therefore, we investigated the cortical morphological changes (cortical thickness, gyrification, sulcal depth, and fractal dimension) of drug-naive BECTS patients with SBM in this study. Also, we analyzed the correlation between abnormal brain regions and age of onset and intelligence quotient (IQ) to explore the possible influence of morphological changes on cognition. We present the following article in accordance with the MDAR reporting checklist (available at http://dx.doi.org/10.21037/atm-205845).

\section{Methods}

\section{Participants}

Twenty-five drug-naive BECTS patients (age: 9.08ะ 1.55 years, range: $7-13$ years, 12 males) and twenty healthy volunteers (age: $9.50 \pm 1.53$ years, range: $7-11$ years, 14 males) were recruited from the Affiliated Hospital of Zunyi Medical University. Inclusion criteria for BECTS patients: (I) BECTS were diagnosed according to the 2010 version diagnostic criteria of the ILAE by intermediate grade pediatricians or higher; (II) not receiving antiepileptic drug treatment before the MRI study; (III) normal routine brain MRI examination; (IV) aged 6-16 years. Exclusion criteria are a history of drug dependence and neurological or psychiatric disorders other than BECTS. Inclusion criteria for the healthy controls (HCs): (I) aged 6-16 years of healthy volunteers; (II) no history of neurological or psychiatric disorders. All BECTS patients completed a neuropsychological assessment. Ages of seizure onset were collected retrospectively from patients' medical files.

The Ethical Committee approved the study of Affiliated Hospital of Zunyi Medical University, and all the informed written consents were obtained from the guardian of participants. All procedures performed in this study involving human participants were in accordance with the Declaration of Helsinki (as revised in 2013).

\section{MRI acquisition}

All participants underwent MRI examinations (3.0T HDxt, GE Healthcare, Milwaukee, WI) after neuropsychological assessment, including a $3 \mathrm{D}-\mathrm{T}_{1}$ acquisition (repetition time $=7.8 \mathrm{~ms}$, echo time $=3.0 \mathrm{~ms}$, inversion time $=450 \mathrm{~ms}$, flip angle $=15^{\circ}$, the field of view $=256 \mathrm{~mm} \times 256 \mathrm{~mm}$, matrix $=256 \times 256$, slice thickness $=1 \mathrm{~mm}$, slices $=256$, scan time $=208$ seconds).

\section{Neuropsychological assessment}

An experienced neuropsychologist conducted the neuropsychological assessment with the Wechsler Intelligence Scale (for Children-Chinese Revised) on the same day of MRI scan, which includes verbal intelligence quotient (VIQ), performance intelligence quotient (PIQ) and full-scale intelligence quotient (FIQ).

\section{Data processing}

All 3D-T $\mathrm{DI}$ data of participants were processed with the Computational Anatomy Toolbox (CAT12) (http://dbm. neuro.uni-jena.de/cat/) within SPM12 with MATLAB R2015a (https://ww2.mathworks.cn/). The procedure includes image format conversion, spatial normalization, brain tissue segmentation, image modulation, and smoothing. The extracted cortical thickness and sulcal depth maps were smoothed with a $15 \mathrm{~mm}$ full-width at half maximum (FWHM) of Gaussian smoothing kernel, 
Table 1 Demographic and clinical characteristics of the BECTS patients and healthy controls

\begin{tabular}{|c|c|c|c|}
\hline Characteristic & BECTS $(n=25)$ & HCs $(n=20)$ & $P$ value \\
\hline Age (years) & $9.08 \pm 1.55$ (range: $7-13$ ) & $9.50 \pm 1.53$ (range: $7-11)$ & 0.37 \\
\hline Handedness & $25 R$ & $20 \mathrm{R}$ & - \\
\hline Education (years) & $3.00 \pm 1.35$ (range: $1-6)$ & $3.60 \pm 1.60$ (range: $1-6)$ & 0.18 \\
\hline Age of onset (years) & $8.56 \pm 1.82$ (range: $5-13)$ & - & - \\
\hline VIQ & $101.84 \pm 12.30$ (range: 81-124) & - & - \\
\hline $\mathrm{PIQ}$ & $104.32 \pm 13.07$ (range: $61-125)$ & - & - \\
\hline $\mathrm{FIQ}$ & $103.28 \pm 11.17$ (range: $76-122)$ & - & - \\
\hline
\end{tabular}

BECTS, benign childhood epilepsy with centrotemporal spikes; HCs, healthy controls; M, male; F, female; R, right; VIQ, verbal intelligence quotient; PIQ, performance intelligence quotient; FIQ, full-scale intelligence quotient.

gyrification, and fractal dimension maps were smoothed with a $25 \mathrm{~mm}$ FWHM of Gaussian smoothing kernel.

\section{Statistical analysis}

Two-sample $t$-tests were performed to assess the differences in age, education, and a chi-square test was used to assess the gender composition between the BECTS and HCs using SPSS (version 18.0; SPSS Inc., Chicago, IL). $\mathrm{P}<0.05$ was considered statistically significant.

Two-sample $t$-tests were carried out to compare the abnormal brain regions of cortical thickness, gyrification, sulcal depth, and fractal dimension between the BECTS patients and $\mathrm{HCs}$ with age and gender as covariates. Familywise error (FWE) was chosen as a correction for multiple comparisons, cluster significance of a $\mathrm{P}<0.05$, cluster size $>30$.

Spearman correlation analyses were used to demonstrate the relationships between cortical characteristics of abnormal brain regions and IQ, which included VIQ, PIQ, and FIQ. $\mathrm{P}<0.05$ was considered statistically significant.

\section{Results}

\section{Demographics and clinical characteristics}

There were no significant differences in age $(\mathrm{P}=0.37)$, gender $(\mathrm{P}=0.13)$ composition, or years in education $(\mathrm{P}=0.18)$ between the BECTS patients and HCs (Table 1).

\section{Cortical thickness}

Compared with HCs, the BECTS patients showed extensive cortical thinning predominantly in bilateral frontal, temporal regions, and limbic system. These included the superior frontal gyrus, rostral middle frontal gyrus, pars orbitalis gyrus, medial orbitofrontal gyrus, precentral gyrus, fusiform gyrus, middle temporal gyrus in the bilateral hemisphere, parahippocampal gyrus, temporal pole, pars opercularis gyrus, caudal middle frontal gyrus, caudal anterior cingulate gyrus, lateral occipital gyrus and insula in the left hemisphere. Also, it included the superior temporal gyrus, paracentral lobule, inferior parietal gyrus, posterior cingulate gyrus, and inferior temporal gyrus in the right hemisphere $(\mathrm{P}<0.05$, FWE corrected, Figure 1).

\section{Cortical gyrification}

The BECTS patients showed significantly increased cortical gyrification in the left hemisphere and partial right hemisphere, including superior frontal gyrus, frontal pole, lingual gyrus, lateral occipital gyrus, isthmus cingulate gyrus, posterior cingulate gyrus, postcentral gyrus, inferior parietal gyrus, supramarginal gyrus, and pars opercularis gyrus in the left hemisphere, inferior temporal gyrus, middle temporal gyrus, pars orbitalis gyrus, superior frontal gyrus in the right hemisphere $(\mathrm{P}<0.05$, FWE corrected, Figure 2). The decreased cortical gyrification was only in the left hemisphere, including the insula, 


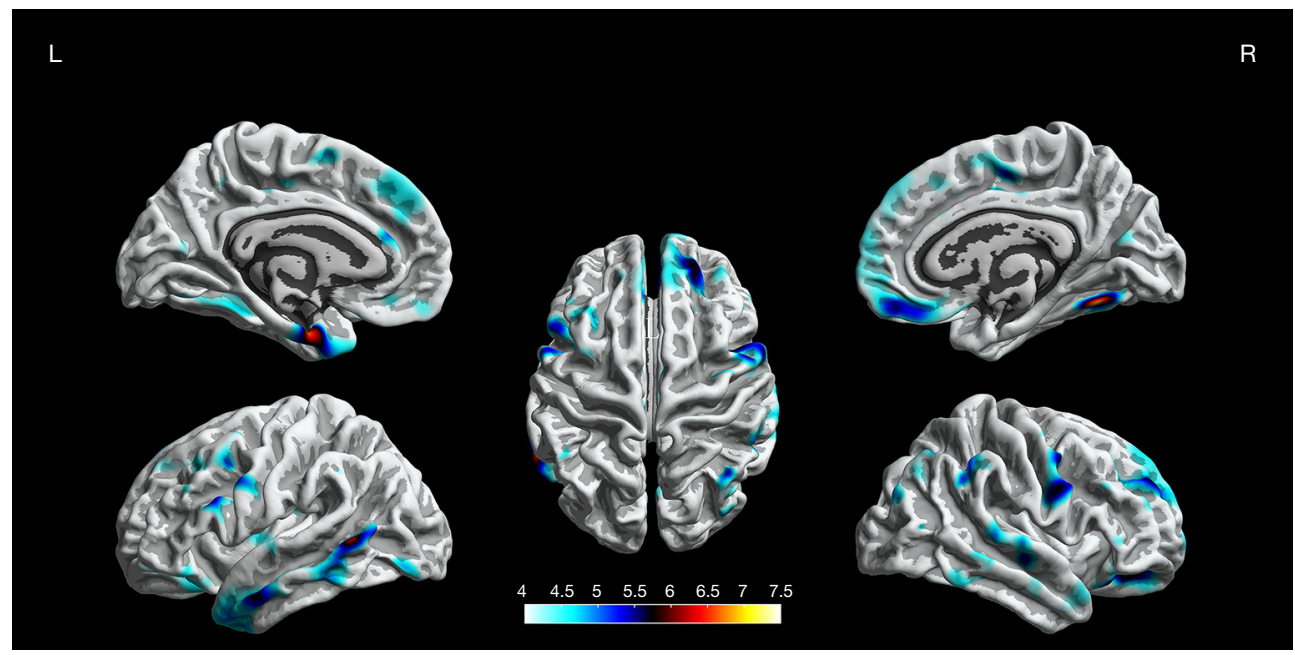

Figure 1 The difference of cortical thickness between the BECTS patients and HCs $(\mathrm{P}<0.05$, FWE corrected). Representative views are shown with a color-coded depiction of abnormalities. Regions of reduced cortical thickness are shown in blue to yellow (color-coded according to $t$ value). BECTS, benign childhood epilepsy with centrotemporal spikes; HCs, healthy controls; FEW, family-wise error.

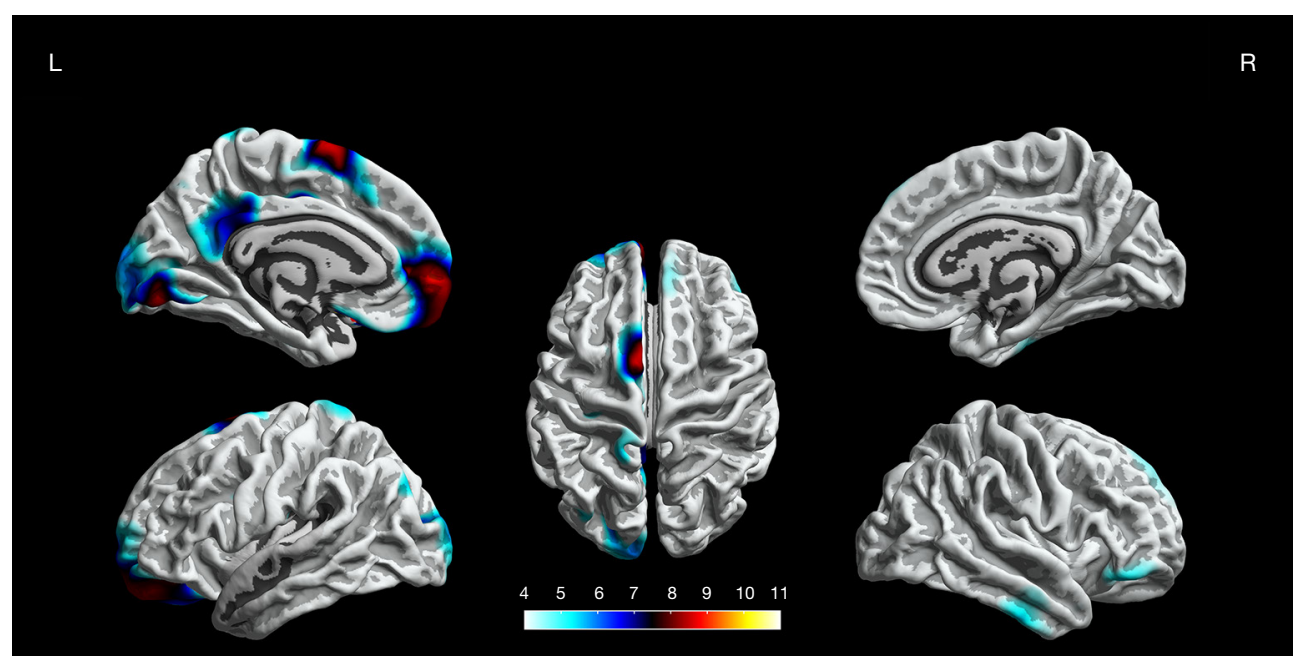

Figure 2 The difference of cortical gyrification between the BECTS patients and HCs $(\mathrm{P}<0.05$, FWE corrected). Representative views are shown with a color-coded depiction of abnormalities. Regions of increased cortical gyrification are shown in blue to yellow (color-coded according to $t$ value). BECTS, benign childhood epilepsy with centrotemporal spikes; HCs, healthy controls; FEW, family-wise error.

pars triangularis gyrus, pars opercularis gyrus, inferior temporal gyrus, lateral occipital gyrus, superior temporal gyrus, middle temporal gyrus and precentral gyrus $(\mathrm{P}<0.05$, FWE corrected, Figure 3).

\section{Sulcal depth}

We found significantly increased sulcal depth in the left fusiform gyrus as the BECTS patients compared to HCs $(\mathrm{P}<0.05$, FWE corrected, Figure 4$)$.

\section{Fractal dimension}

There are no statistically significant differences in fractal dimension between the BECTS patients and HCs $(\mathrm{P}<0.05$, FWE corrected). 


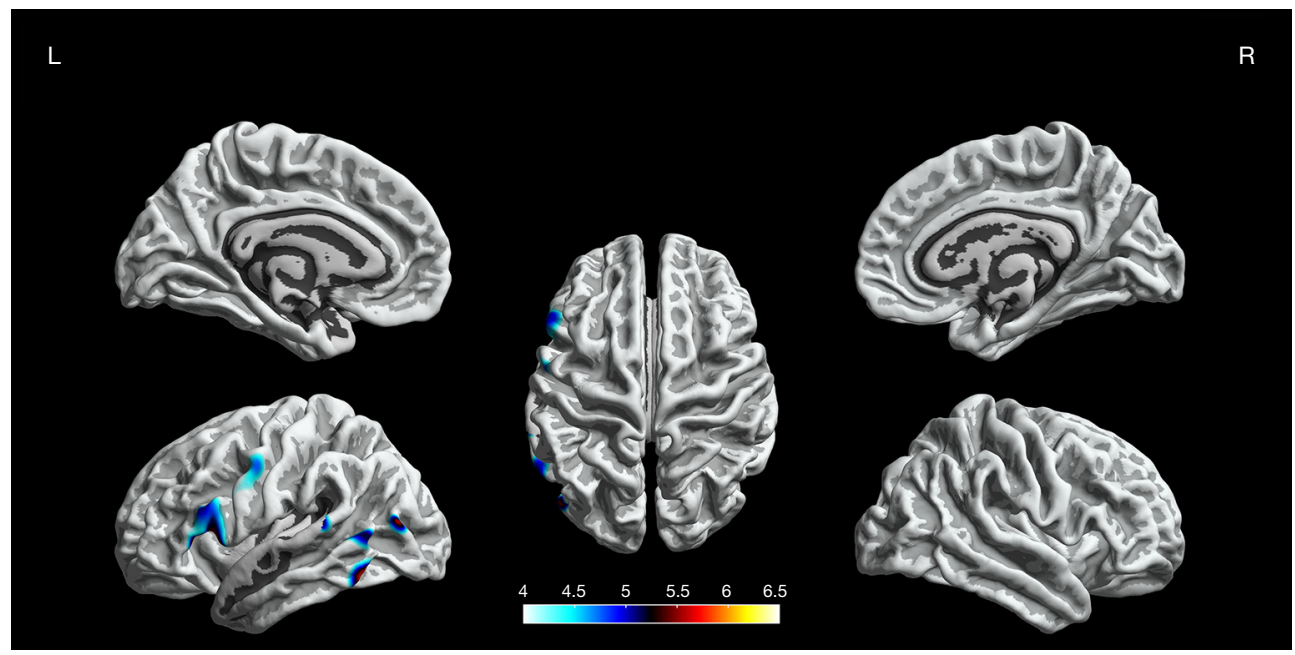

Figure 3 The difference of cortical gyrification between the BECTS patients and HCs $(\mathrm{P}<0.05$, FWE corrected). Representative views are shown with a color-coded depiction of abnormalities. Regions of decreased cortical gyrification are shown in blue to yellow (color-coded according to $t$ value). BECTS, benign childhood epilepsy with centrotemporal spikes; HCs, healthy controls; FEW, family-wise error.

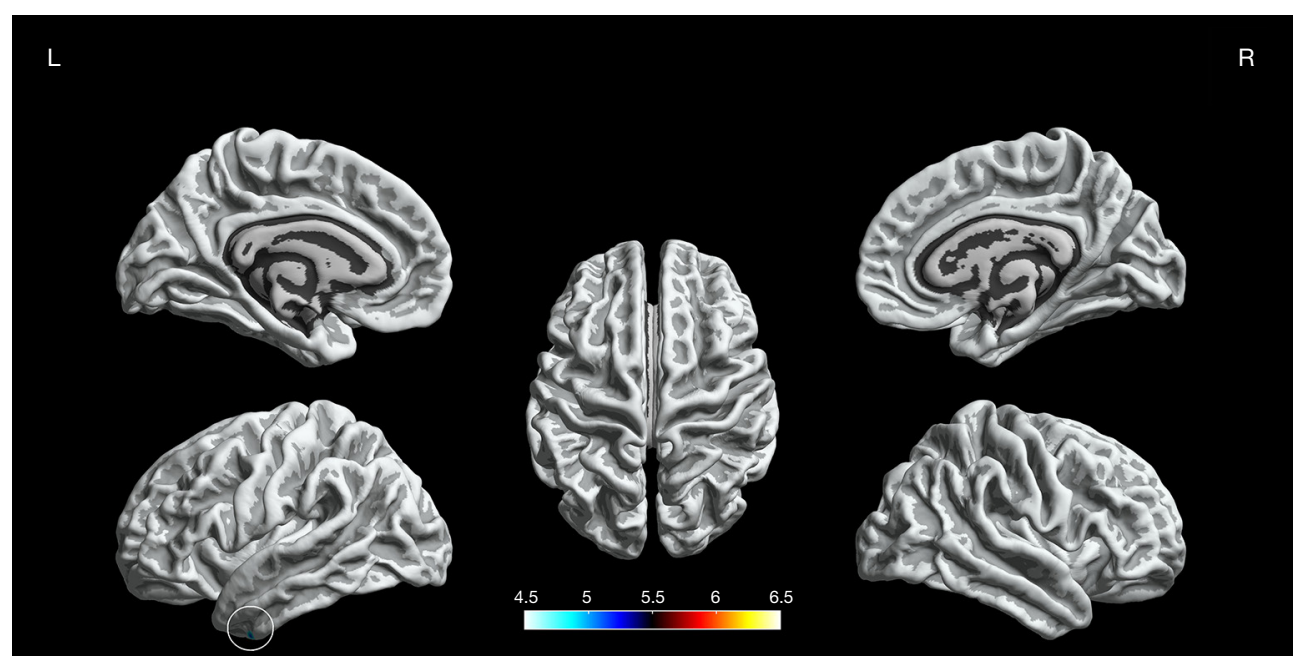

Figure 4 The difference of sulcal depth between the BECTS patients and HCs $(\mathrm{P}<0.05$, FWE corrected). The representative view is shown with a color-coded depiction of abnormalities. Regions of increased sulcal depth are shown in blue within the circle (color-coded according to $t$ value). BECTS, benign childhood epilepsy with centrotemporal spikes; HCs, healthy controls; FEW, family-wise error.

\section{Correlation}

Correlation analysis revealed the negative correlation between age of onset and cortical thickness in the right precentral gyrus $\left(r_{s}=-0.495, \mathrm{P}=0.011\right.$, Figure 5$)$, age of onset and cortical gyrification in the left inferior parietal gyrus $\left(r_{s}=-0.523, \mathrm{P}=0.007\right.$, Figure 6), VIQ and cortical gyrification in the left supramarginal gyrus of BECTS patients $\left(r_{s}=-0.455, \mathrm{P}=0.022\right.$, Figure 7$)$.

\section{Discussion}

In the current study, we investigated differences in cortical thickness, gyrification, sulcal depth, and fractal dimension of drug-naive BECTS patients compared to HCs with SBM. We found aberrant morphology in thickness, gyrification, and sulcal depth, but the fractal dimension showed no difference. Specifically, the BECTS patients showed extensive cortical thinning predominantly in 


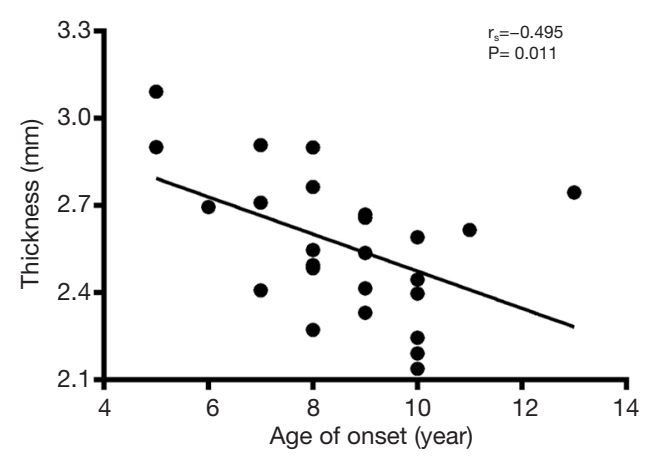

Figure 5 Scatter plots of the mean cortical thickness of the clusters in the right precentral gyrus, which were negatively correlated with age of onset in the BECTS patients. BECTS, benign childhood epilepsy with centrotemporal spikes.

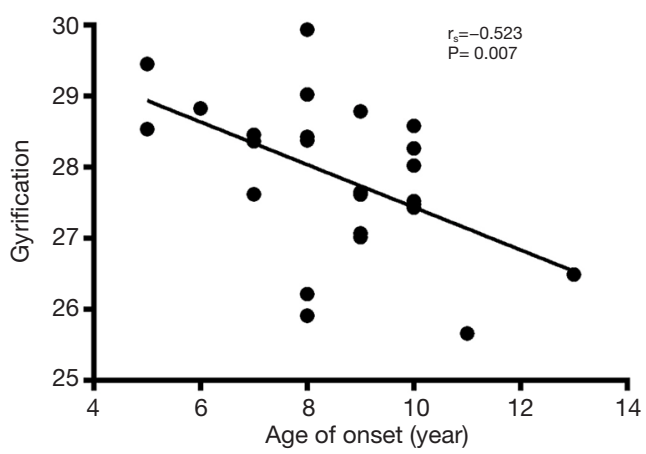

Figure 6 Scatter plots of the mean cortical gyrification of the clusters in the left inferior parietal gyrus, which were negatively correlated with age of onset in the BECTS patients. BECTS, benign childhood epilepsy with centrotemporal spikes.

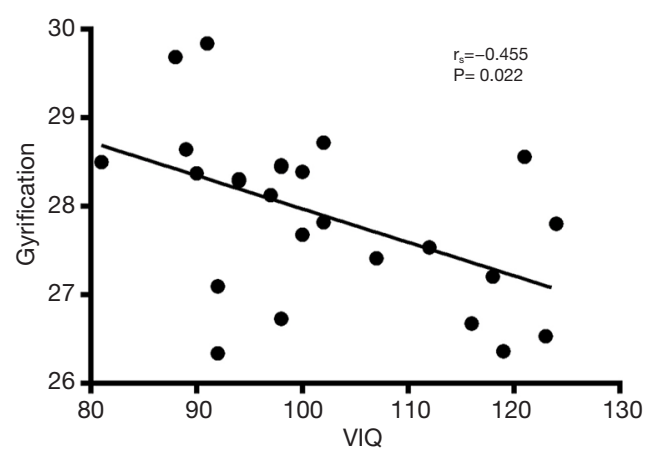

Figure 7 Scatter plots of the mean cortical gyrification of the clusters in the left supramarginal gyrus negatively correlated with VIQ in the BECTS patients. VIQ, verbal intelligence quotient; BECTS, benign childhood epilepsy with centrotemporal spikes. bilateral frontal, temporal regions and limbic system, increased cortical gyrification in the left hemisphere and partial right hemisphere, decreased cortical gyrification was only in the left hemisphere and increased sulcal depth in the left fusiform gyrus. Also, we observed negative correlations between cortical thickness/cortical gyrification and age of onset, cortical gyrification and VIQ of BECTS patients.

Previous studies have shown abnormal cortical thickness in different brain regions of BECTS patients, in which thicker cortex is predominant, and only a few studies have found cortical thinning (5,6,11-13). We also found BECTS patients with extensive cortical thinning in bilateral frontal, temporal regions, and limbic system. Evidence suggests that the development of the human cortex is dynamic and expansion-renormalization $(18,19)$. Learning induces cortical thickening in a brief time. Then inefficient connections were pruned to stabilize neural circuitry, including reducing dendritic arborizations and eliminating axonal projections selectively, etc., which lead to cortical thinning (19-21). Added studies showed that cortical development deviates from the normal trajectory in BECTS. For example, Overvliet et al. (6) revealed cerebral cortical thinning in the advance of BECTS patients. GarciaRamos et al. (12) observed different brain regions of cortical thickening and thinning during the two years after the onset of BECTS, while the HCs showed extensive cortical thinning in bilateral cerebral hemispheres. In a word, cortical change is abnormal in BECTS patients.

As shown in some studies, BECTS patients existed cortical thickening in some brain regions $(5,11)$. Other studies observed the increased volume of bilateral putamen in BECTS patients and the negative correlation between the age of epilepsy onset and volume in bilateral putamen $(5,10,12,22)$. The more significant the area the putamen accompanies, the better executive function was observed and vice versa (22). It is considered that high performance is an adaptive change, which is beneficial to survival. Thus, cortical thickening of BECTS patients was due to epileptiform discharge caused compensatory hyperplasia in the discharge regions or adjacent areas. However, we only found extensive cortical thinning and no significant area of thickening in BECTS patients that may be associated with short duration time or distinct stages of brain development.

Also, we found increased cortical gyrification in the left hemisphere and partial right hemisphere and decreased cortical gyrification in the left hemisphere. Further, we found the BECTS patients' neuropsychological scores were under or near normal, which was observed in some 
studies $(6,10,12,23)$. As we all know, BECTS has language impairment and has indeed been recognized to belong to the epilepsy aphasia spectrum $(24,25)$. We observed a negative correlation between VIQ and cortical gyrification in the left supramarginal gyrus of BECTS patients. Therefore, the reason we found the change of cortical gyrification mainly located in the left hemisphere that same as the study in Jiang et al. (23) is probably the laterality of cognitive functions or regions with language dominance.

In the present and almost all previous studies about BECTS showed abnormalities of cortical thickness and gyrification in different brain regions that the Rolandic region was dominated but not confined to it $(5,6,11-13,23)$. Brain network abnormalities of gray matter and white matter exceed the Rolandic region that even extends from the ipsilateral hemisphere to the contralateral hemisphere may be explained cortical abnormalities beyond the seizure onset zone, which probably means the propagation of epileptiform discharges through networks $(26,27)$. In this study, we reported increased cortical gyrification in regions that avoided left temporal lobes and partial frontal lobes, but these regions were Rolandic and peripheral components that showed decreased cortical gyrification. Furthermore, our results showed that cortical gyrification was negatively correlated with intelligence, which is opposite to earlier studies in healthy subjects $(19,28,29)$. The negative correlation between cortical gyrification and executive function was observed in schizophrenia (30). We infer pathology that leads to corticocortical connectivity decreased, interrupted, and then restored. Epileptic discharge in the Rolandic region may cause severe damage in this area and peripheral regions so that they do not restore.

We found abnormal cortical thickness and gyrification in a language center, including middle frontal gyrus, inferior frontal gyrus, middle temporal gyrus, and inferior temporal gyrus, which may be responsible for apparent language dysfunction in BECTS patients. It is well-known that the language center is not independent but interconnected with other brain regions. Previous researches also revealed reduced connectivity between the Rolandic region and Broca's area associated with poor language in BECTS patients, which means an impaired interplay between motor and language networks as well as the correlation of epileptiform activity and language impairment $(31,32)$. Another study found BECTS patients with abnormal connectivity from Broca's area to the prefrontal cortex, lingual gyrus, hippocampus, etc. by granger causality analysis (33). Considering the above studies, we found widespread cortical thinning and abnormal gyrification not limited to language centers that may handle language dysfunction in BECTS patients.

What should not be neglected is we found abnormal cortical thickness and gyrification in the limbic system, especially in the cingulate gyrus. The limbic system plays a vital role in memory, behavior, and emotion, etc. (34). Attention deficit hyperactivity disorder (ADHD)-related symptoms interpreted by abnormal default mode network (DMN) was accepted, and posterior cingulate gyrus served as a crucial region in DMN $(35,36)$. The higher incidence of ADHD in BECTS patients may be associated with this structural change, although BECTS patients were not suspected of ADHD by pediatricians in this study $(5,37,38)$. Further, posterior cingulate gyrus involved in multiple networks, including the executive control network, memory network, etc., a critical member in the "rich club" to facilitate communication in global information integration $(39,40)$. Thus, morphological abnormalities in the posterior cingulate gyrus may play a role in the cognitive dysfunction of BECTS patients, particularly in attention, memory, and executive function.

Increased sulcal depth in the left fusiform gyrus was found in BECTS patients. The change in sulcal depth resulting from cortico-cortical connections was supposed in previous studies, which can reflect cortical folding $(41,42)$. However, there is no significant cortical gyrification reported in the left fusiform gyrus; the change of sulcal depth serves as a supplementary method. The decreased sulcal depth was observed in schizophrenia and Alzheimer's disease, which decreased with disease progression $(41,42)$. The decreased sulcal depth was observed with age in healthy adults (43). Therefore, the increased sulcal depth may not be the wrong way. The fractal dimension was used to estimate the complexity of the brain, which reflects neurodevelopment (44). Abnormal fractal dimension was observed in neuropsychiatric diseases, including premanifested Huntington's disease, frontotemporal dementia, etc. $(45,46)$. In our study, there are no statistically significant differences in the fractal dimension that might be due to less prominence of neural changes or small sample sizes.

We found cortical thickness in the right precentral gyrus and cortical gyrification in the left inferior parietal gyrus were significantly negatively correlated with the age of onset. Also, it was determined that cortical gyrification in the left supramarginal gyrus was significantly negatively correlated with VIQ in BECTS patients. The change of 
cortical thickness is dynamic, and intelligence is more related to the magnitude over time of cortical thickness changes during development than to cortical thickness per se (47). However, our results showed that cortical gyrification was correlated with intelligence, which was in line with earlier studies $(19,28,29)$. For language function, studies presented a younger age of epilepsy onset with better prognosis through recombination, but it is also possible to lead severe damage due to early epileptic discharge, especially in the critical period of language formation $(25,48,49)$. There is no relationship between VIQ and the age of onset in our study. Therefore, it is unclear whether younger or older age of onset will be suitable for the development of BECTS patients.

There are several limitations to this study. First, although neuropsychological assessment was employed in BECTS patients, the specific neuropsychological examination on the cognitive function was absent, including language, attention, and memory. Then, healthy controls lacked a neuropsychological assessment, but we propose that these individuals had better profiles due to this. Finally, as a crosssectional study with small subject numbers, large sample size and longitudinal follow-up are necessary to explain dynamic changes of cortical morphology and the causality with cognitive function.

\section{Conclusions}

This study reveals aberrant cortical thickness, cortical gyrification, and sulcal depth of BECTS in areas related to cognitive functions including language, attention and memory, and the correlation between some brain regions and VIQ and age of onset, which provides a new biomarker for the study of cognitive function in BECTS.

\section{Acknowledgments}

Funding: This work was supported by the National Natural Science Foundation of China (grant No. 81760309 , 81960312), Science and Technology Foundation of Health and Family Planning Commission of Guizhou Province (grant No. gzwjkj2017-1-013), Science and Technology Foundation of Guizhou Province \{grant No. Qiankeheping tairencai[2017]5620\}, Innovative Research Team of Medical Imaging Center in Higher Education Institutions of Guizhou Province \{grant No. Qianjiaoherencaituandui[2014]37\}, SZU Top Ranking Project, 352 Shenzhen University (860/000002100108), Shenzhen-Hong Kong Institute of Brain Science-353 Shenzhen Fundamental Research Institutions (2019SHIBS0003).

\section{Footnote}

Reporting Checklist: The authors have completed the MDAR reporting checklist. Available at http://dx.doi.org/10.21037/ atm-20-5845

Data Sharing Statement: Available at http://dx.doi. org/10.21037/atm-20-5845

Conflicts of Interest: All authors have completed the ICMJE uniform disclosure form (available at http://dx.doi. org/10.21037/atm-20-5845). The authors have no conflicts of interest to declare.

Ethical Statement: The authors are accountable for all aspects of the work in ensuring that questions related to the accuracy or integrity of any part of the work are appropriately investigated and resolved. The Ethical Committee approved the study of the Affiliated Hospital of Zunyi Medical University (No. 1-047), and all the informed written consents were obtained from parents of participants. All procedures performed in this study involving human participants were in accordance with the Declaration of Helsinki (as revised in 2013).

Open Access Statement: This is an Open Access article distributed in accordance with the Creative Commons Attribution-NonCommercial-NoDerivs 4.0 International License (CC BY-NC-ND 4.0), which permits the noncommercial replication and distribution of the article with the strict proviso that no changes or edits are made and the original work is properly cited (including links to both the formal publication through the relevant DOI and the license). See: https://creativecommons.org/licenses/by-nc-nd/4.0/.

\section{References}

1. Dryżałowski P, Jóźwiak S, Franckiewicz M, et al. Benign epilepsy with centrotemporal spikes - Current concepts of diagnosis and treatment. Neurol Neurochir Pol 2018;52:677-89.

2. Scheffer IE, Berkovic S, Capovilla G, et al. ILAE classification of the epilepsies: Position paper of the ILAE Commission for Classification and Terminology. Epilepsia 2017;58:512-21. 
3. Liu MJ, Su XJ, Shi XY, et al. Clinical features of benign epilepsy of childhood with centrotemporal spikes in chinese children. Medicine (Baltimore) 2017;96:e5623.

4. Choi HS, Chung YG, Choi SA, et al. Electroencephalographic Resting-State Functional Connectivity of Benign Epilepsy with Centrotemporal Spikes. J Clin Neurol 2019;15:211-20.

5. Kim EH, Yum MS, Shim WH, et al. Structural abnormalities in benign childhood epilepsy with centrotemporal spikes (BCECTS). Seizure 2015;27:40-6.

6. Overvliet GM, Besseling RM, Jansen JF, et al. Early onset of cortical thinning in children with rolandic epilepsy. Neuroimage Clin 2013;2:434-9.

7. Teixeira J, Santos ME. Language skills in children with benign childhood epilepsy with centrotemporal spikes: A systematic review. Epilepsy Behav 2018;84:15-21.

8. Wickens S, Bowden SC, D'Souza W. Cognitive functioning in children with self-limited epilepsy with centrotemporal spikes: A systematic review and metaanalysis. Epilepsia 2017;58:1673-85.

9. Verrotti A, Filippini M, Matricardi S, et al. Memory impairment and Benign Epilepsy with centrotemporal spike (BECTS): a growing suspicion. Brain Cogn 2014;84:123-31.

10. Luo C, Zhang Y, Cao W, et al. Altered Structural and Functional Feature of Striato-Cortical Circuit in Benign Epilepsy with Centrotemporal Spikes. Int J Neural Syst 2015;25:1550027.

11. Pardoe HR, Berg AT, Archer JS, Fulbright RK, Jackson GD. A neurodevelopmental basis for BECTS: evidence from structural MRI. Epilepsy Res 2013;105:133-9.

12. Garcia-Ramos C, Jackson DC, Lin JJ, et al. Cognition and brain development in children with benign epilepsy with centrotemporal spikes. Epilepsia 2015;56:1615-22.

13. Karalok ZS, Öztürk Z, Gunes A. Cortical thinning in benign epilepsy with centrotemporal spikes (BECTS) with or without attention-deficit/hyperactivity (ADHD). J Clin Neurosci 2019;68:123-7.

14. Spalthoff R, Gaser C, Nenadić I. Altered gyrification in schizophrenia and its relation to other morphometric markers. Schizophr Res 2018;202:195-202.

15. Hwang G, Dabbs K, Conant L, et al. Cognitive slowing and its underlying neurobiology in temporal lobe epilepsy. Cortex 2019;117:41-52.

16. Li M, Hua K, Li S, et al. Cortical morphology of chronic users of codeine-containing cough syrups: association with sulcal depth, gyrification, and cortical thickness. Eur Radiol 2019;29:5901-9.
17. Ruiz de Miras J, Costumero V, Belloch V, et al. Complexity analysis of cortical surface detects changes in future Alzheimer's disease converters. Hum Brain Mapp 2017;38:5905-18.

18. Muftuler LT, Davis EP, Buss C, et al. Cortical and subcortical changes in typically developing preadolescent children. Brain Res 2011;1399:15-24.

19. Tadayon E, Pascual-Leone A, Santarnecchi E. Differential Contribution of Cortical Thickness, Surface Area, and Gyrification to Fluid and Crystallized Intelligence. Cereb Cortex 2020;30:215-25.

20. Wenger E, Brozzoli C, Lindenberger U, et al. Expansion and Renormalization of Human Brain Structure During Skill Acquisition. Trends Cogn Sci 2017;21:930-9.

21. Wenger E, Kühn S, Verrel J, et al. Repeated Structural Imaging Reveals Nonlinear Progression of ExperienceDependent Volume Changes in Human Motor Cortex. Cereb Cortex 2017;27:2911-25.

22. Lin JJ, Riley JD, Hsu DA, et al. Striatal hypertrophy and its cognitive effects in new-onset benign epilepsy with centrotemporal spikes. Epilepsia 2012;53:677-85.

23. Jiang L, Zhang T, Lv F, et al. Structural Covariance Network of Cortical Gyrification in Benign Childhood Epilepsy with Centrotemporal Spikes. Front Neurol 2018;9:10.

24. Roulet-Perez E, Mayor C. Childhood epilepsy with centro-temporal spikes (rolandic epilepsy) and written language. Dev Med Child Neurol 2018;60:219.

25. Jackson DC, Jones JE, Hsu DA, et al. Language function in childhood idiopathic epilepsy syndromes. Brain Lang 2019;193:4-9.

26. Jiang Y, Song L, Li X, et al. Dysfunctional whitematter networks in medicated and unmedicated benign epilepsy with centrotemporal spikes. Hum Brain Mapp 2019;40:3113-24.

27. Adebimpe A, Bourel-Ponchel E, Wallois F. Identifying neural drivers of benign childhood epilepsy with centrotemporal spikes. Neuroimage Clin 2017;17:739-50.

28. Gregory MD, Kippenhan JS, Dickinson D, et al. Regional Variations in Brain Gyrification Are Associated with General Cognitive Ability in Humans. Curr Biol 2016;26:1301-5.

29. Gautam P, Anstey KJ, Wen W, et al. Cortical gyrification and its relationships with cortical volume, cortical thickness, and cognitive performance in healthy mid-life adults. Behav Brain Res 2015;287:331-9.

30. Sasabayashi D, Takayanagi Y, Nishiyama S, et al. Increased Frontal Gyrification Negatively Correlates with Executive 
Function in Patients with First-Episode Schizophrenia. Cereb Cortex 2017;27:2686-94.

31. Besseling RM, Jansen JF, Overvliet GM, et al. Reduced functional integration of the sensorimotor and language network in rolandic epilepsy. Neuroimage Clin 2013;2:239-46.

32. Besseling RM, Overvliet GM, Jansen JF, et al. Aberrant functional connectivity between motor and language networks in rolandic epilepsy. Epilepsy Res 2013;107:253-62.

33. Chen S, Fang J, An D, et al. The focal alteration and causal connectivity in children with new-onset benign epilepsy with centrotemporal spikes. Sci Rep 2018;8:5689.

34. Catani M, Dell'acqua F, Thiebaut de Schotten M. A revised limbic system model for memory, emotion and behaviour. Neurosci Biobehav Rev 2013;37:1724-37.

35. Zepf FD, Bubenzer-Busch S, Runions KC, et al. Functional connectivity of the vigilant-attention network in children and adolescents with attention-deficit/ hyperactivity disorder. Brain Cogn 2019;131:56-65.

36. Xiao F, Li L, An D, et al. Altered attention networks in benign childhood epilepsy with centrotemporal spikes (BECTS): A resting-state fMRI study. Epilepsy Behav 2015;45:234-41.

37. Lima EM, Rzezak P, Dos Santos B, et al. The relevance of attention deficit hyperactivity disorder in self-limited childhood epilepsy with centrotemporal spikes. Epilepsy Behav 2018;82:164-9.

38. Kim EH, Yum MS, Kim HW, et al. Attention-deficit/ hyperactivity disorder and attention impairment in children with benign childhood epilepsy with centrotemporal spikes. Epilepsy Behav 2014;37:54-8.

39. Lord AR, Li M, Demenescu LR, et al. Richness in Functional Connectivity Depends on the Neuronal Integrity within the Posterior Cingulate Cortex. Front Neurosci 2017;11:184.

40. Busler JN, Yanes JA, Bird RT, et al. Differential functional patterns of the human posterior cingulate cortex during activation and deactivation: a meta-analytic connectivity model. Exp Brain Res 2019;237:2367-85.

41. Lyu I, Kang H, Woodward ND, et al. Sulcal Depthbased Cortical Shape Analysis in Normal Healthy Control and Schizophrenia Groups. Proc SPIE Int Soc Opt Eng 2018;10574:1057402.

42. Im K, Lee JM, Seo SW, et al. Sulcal morphology changes and their relationship with cortical thickness and gyral white matter volume in mild cognitive impairment and Alzheimer's disease. Neuroimage 2008;43:103-13.

43. Kochunov P, Mangin JF, Coyle T, et al. Age-related morphology trends of cortical sulci. Hum Brain Mapp 2005;26:210-20.

44. Di Ieva A, Esteban FJ, Grizzi F, et al. Fractals in the neurosciences, Part II: clinical applications and future perspectives. Neuroscientist 2015;21:30-43.

45. Sheelakumari R, Rajagopalan V, Chandran A, et al. Quantitative analysis of grey matter degeneration in FTD patients using fractal dimension analysis. Brain Imaging Behav 2018;12:1221-8.

46. Kubera KM, Schmitgen MM, Hirjak D, et al. Cortical neurodevelopment in pre-manifest Huntington's disease. Neuroimage Clin 2019;23:101913.

47. Schnack HG, van Haren NE, Brouwer RM, et al. Changes in thickness and surface area of the human cortex and their relationship with intelligence. Cereb Cortex 2015;25:1608-17.

48. Currie NK, Lew AR, Palmer TM, et al. Reading comprehension difficulties in children with rolandic epilepsy. Dev Med Child Neurol 2018;60:275-82.

49. Baumer FM, Cardon AL, Porter BE. Language Dysfunction in Pediatric Epilepsy. J Pediatr 2018;194:13-21.

(English Language Editor: J. Chapnick)
Cite this article as: Li Z, Zhang J, Wang F, Yang Y, Hu J, Li Q, Tian M, Li T, Huang B, Liu H, Zhang T. Surface-based morphometry study of the brain in benign childhood epilepsy with centrotemporal spikes. Ann Transl Med 2020;8(18):1150. doi: 10.21037/atm-20-5845 